\title{
Digital Video Watermarking for Educational Video Broadcasting and Monitoring Application
}

\author{
Dixa \\ SVIT, Vasad \\ Gujarat, India
}

\author{
Manoj Pandya \\ BISAG, Gandhinagar \\ Gujarat, India
}

\author{
Nehal Chitaliya \\ SVIT, Vasad \\ Gujarat, India
}

\author{
M.B. Potdar \\ BISAG,Gandhinagar \\ Gujarat, India
}

\begin{abstract}
The Proposed work for digital video watermarking is mainly belonging to distance or vocational education industries for the copyright protection of their recorded and broadcasted lecture series from any illegal commercial use. It provides two level security to recorded \& broadcasted educational videos. One level of security is provided by visible serial key and other is provided by embedding invisible watermark logo. Invisible watermark is embedded within the frame at which scene change is detected using Modified LSB method. Invisible watermark is used when visible watermark is removed from frames. Experimental results show that execution time require for embedding the watermark is very less which enables it to implement in real time and robust against various video and image processing attacks, noise attacks.
\end{abstract}

\section{Keywords}

Video Broadcasting, DWT, LSB, PSNR, SF, Perceptibility

\section{INTRODUCTION}

With millions of Web sites flooding over the Internet, it becomes easy for online pirates to copy-paste and to modify the information, images, video and audio. The central goal for technical researchers in Digital Watermarking application is to address the copyright infringement issue by developing the algorithm which provides evidence against any illegal attempt to modify the original content and also monitors and tracks how the original content is used over the internet [1]-[6]. Educational industries which provides the Vocational/ Distance education needs to record and deliver the subject lectures in order to provide the education at student's door step. This cost a huge as it needs to hire qualified teacher for delivering lectures as well as good set up of recording studios is also mandatory. Recorded lectures can be delivered to student by uploading it over internet or by broadcasting it on any educational channel or by distributing the DVD of recorded lectures. As such video can be easily modified, distributed and used by any individual for their commercial use, some means of protection over video modification is required [7]. Film industries also need such protection over video piracy problem. Invisible video

Watermarking is one of the best solutions to overcome such problems. For authentication purpose, watermark (company's identity) is embedded invisibly within the original video which works as an evidence for any illegal attempt to manipulate the recoded video.

Digital watermarking for video broadcasting and monitoring application

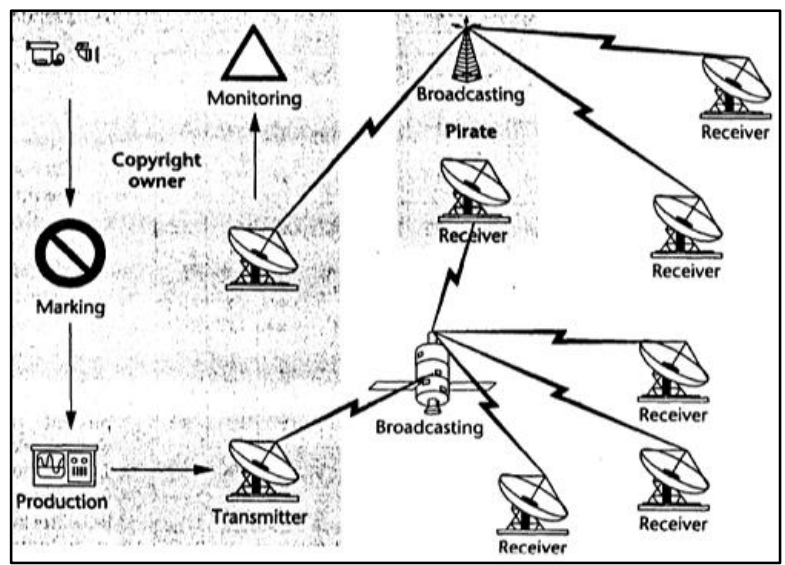

Figure 1: Digital Video Watermarking for Broadcasting and Monitoring Application [2]

Figure 1 shows the real time scenario of video watermarking for broadcasting and monitoring application. As shown, before production, watermark is embedded into video which is being broadcasted. At the receiver side, watermark is changed by hackers which indicated manipulation of video. Such pirated videos are detected by video broadcasting and monitoring application. Digital Video Watermarking can be classified by various types. According to perceptivity, it can be visible or invisible. Visible watermarking is mostly used to declare the identity of owner. It is widely used in video editors which are freely available over the internet for video editing. After editing any video/audio/image, software creates the visible watermark. Invisible watermarking is mainly used for authentication purpose.

Spatial Domain Techniques directly deals with pixel values to embed the watermark. Frequency domain technique deals with the transform coefficients to embed the watermark [8][12]. Widely used spatial domain technique is Least Significant Bit method (LSB) in which LSBs of original image gets replaced with MSB (Most Significant Bit) of watermark logo image [13]-[18]. Though it is easy to implement, it is not robust against various attacks. The algorithm embeds 8 bit-plane gray scale image into different scene of a video sequence by selecting some of the luminous value in video pictures. Scene change detection based watermarking method avoids the process of embedding watermark on each frame of video. Frequency Domain Technique includes various transforms like DCT (Discrete Cosine Transform), DWT (Discrete Wavelet Transform), DFT (Discrete Fourier Transform).Extensive research has been carried out for multidirectional transforms like Wavelet, Contourlet, Ridgelet.

For the proposed method (i) original video can be of any size i.e. not mandatory be squared video (ii) RGB watermark logo 
can be used (iii) Watermark detection algorithm doesn't require original video (vi) Execution time required for watermark embedding is less as compared to other method Which enables it to use in real time. The reminder of this paper includes proposed method, experimental results followed by conclusion and future scope.

\section{PROPOSED WATERMARKING TECHNIQUE}

Every video contains number of continuous identical video frames which is known as video shot. Number of identical frames are larger for slow motion videos (recorded video lecture series which includes rarely two or three different scene) as compared to fast motion entertainment video. To avoid the process on each video frame, one frame is selected per video shot to embed the watermark. Scene change detection based on statistical parameter MSE (Mean square error) is used to identify the frame at which sudden change is detected.

\subsection{Generalized Model for Video Water- marking Algorithm}

Figure 2 describes overall video watermarking process. It consist of four major steps. Visible watermark insertion, Invisible watermark embedding, Attacks and watermark detection. Video frame extraction algorithm process on original video and extract all frames of video. Scene change detection algorithm selects the frame for watermark embedding from each video shot. Watermark Embedding Algorithm embeds the watermark on selected frame. Watermark extraction algorithm detects the embedded watermark.

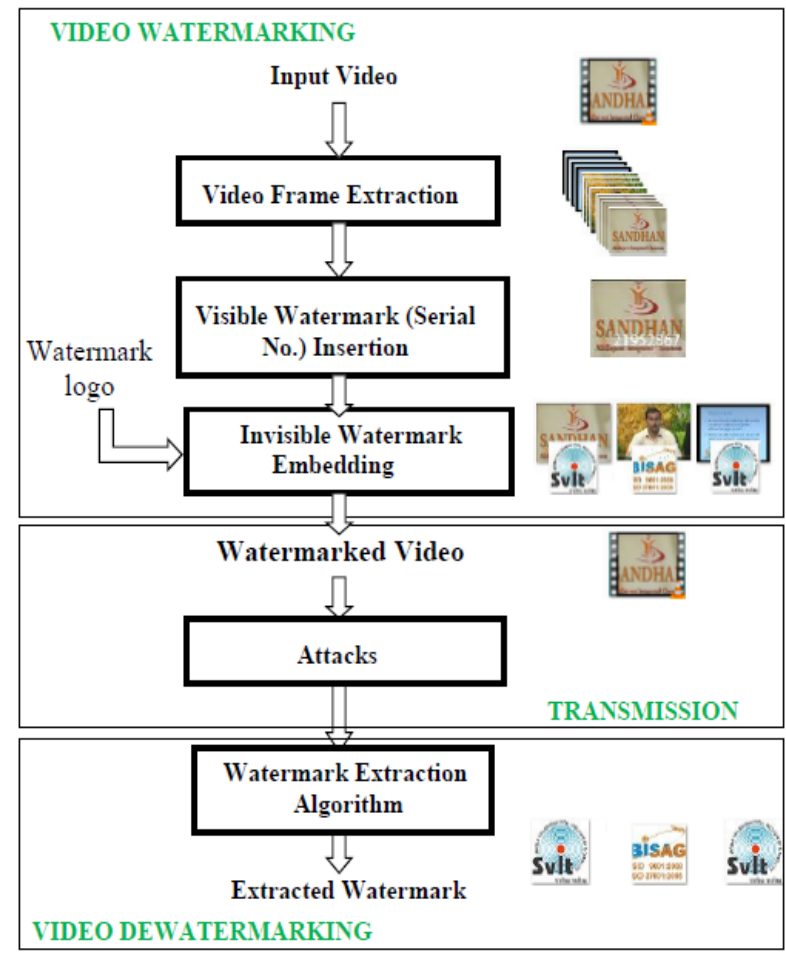

Figure 2 : Generalized model for Video Watermarking Process

\subsubsection{Visible Watermark Insertion}

After extracting frames of video, select bunch of few frames to insert visible watermark. The visible watermark is unique 8 digit serial key. As it is inserted for few frames, it is not visible in whole video and difficult to detect by normal video viewer but it helps to detect any modification done on video. Hence, Visible Serial key provides first level authentication to video. If some attacks are performed and this serial key is removed, second level authentication is provided to video using Invisible watermark embedding.

\subsubsection{Invisible Watermark Embedding Algorithm}

Invisible video watermarking can be describe in two parts, Scene change detection and Watermark embedding algorithm.

\section{Scene Change Detection Algorithm}

Every video contains bundles of identical frames which is known as video shot. Every video has number of such video shots. In Educational recorded videos are slow motion videos having very few scene changes. So, Video shots extracted for educational lecture videos are less as compared to other type of videos. This can be taken as advantage to avoid the process on each and every frame of video.

As shown in Figure 3, Scene change detection algorithm detects first frame of every video shot to embed the watermark. To detect the frame, each frame is converted into grayscale and difference between two consecutive frames is obtained. If it is greater than some threshold value, it indicates the scene change and current frame is selected to embed the watermark.

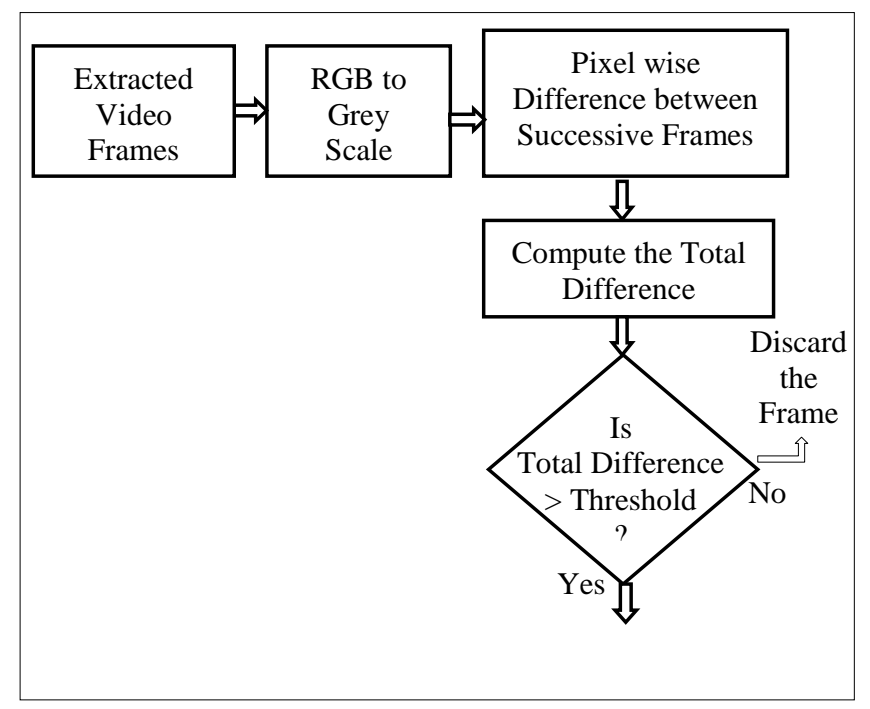

Figure 3: Scene Change Detection Algorithm 


\section{Watermark Embedding Algorithm}

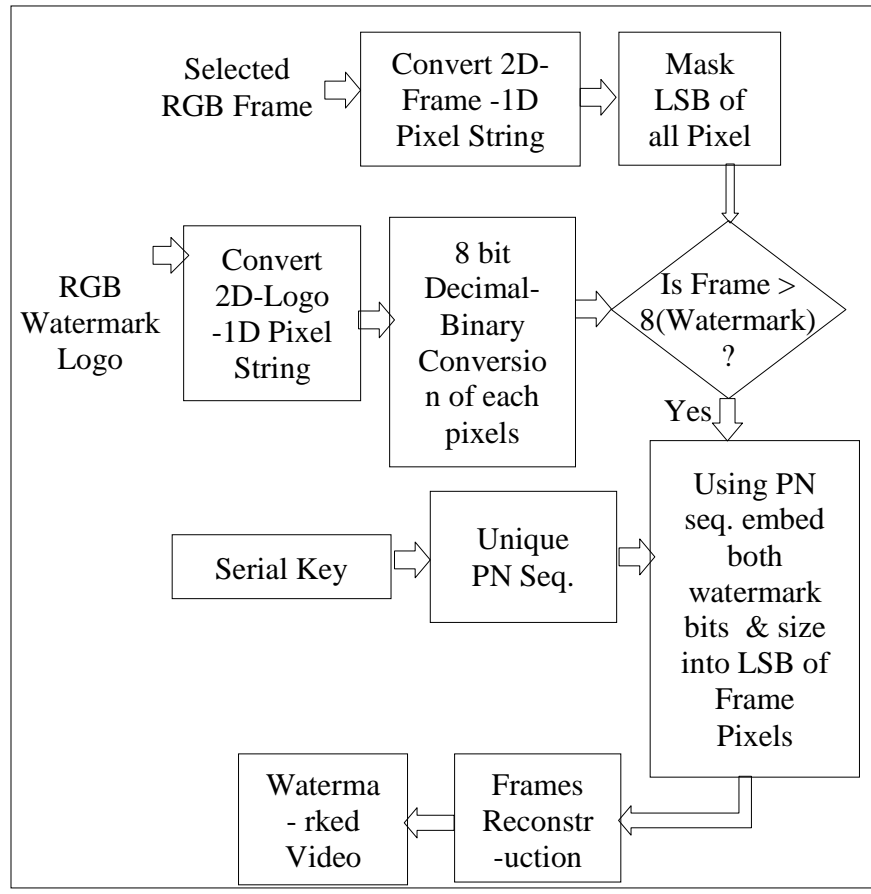

Figure 4: Watermark Embedding Algorithm

Figure 4 describes watermark embedding algorithm in detail. Selected RGB frame is converted into 1D pixel string. Then LSB of all pixels are masked. Same procedure is repeated for RGB watermark logo. Using randomly generated visible serial key, pixel of watermark logo is embedded into LSB of each pixels of original frame. Pixel string is converted back into 2D watermarked frame.

\subsubsection{Watermark Extraction Algorithm}

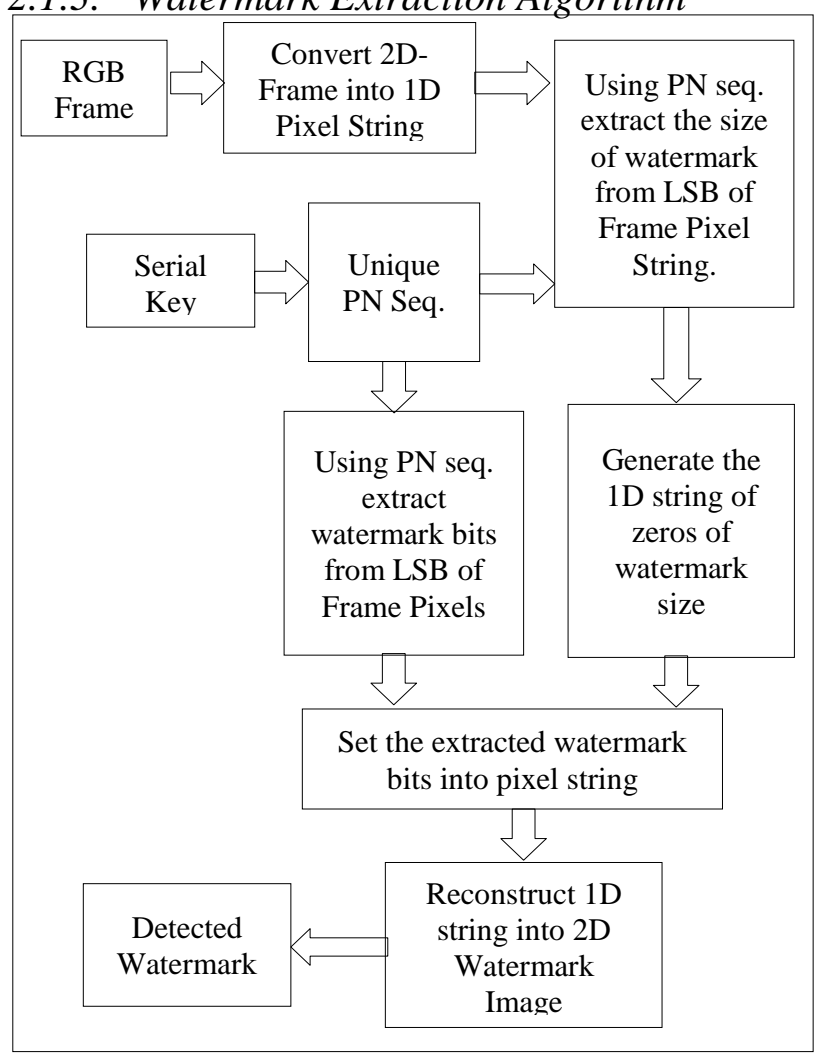

Figure 5: Video Watermark Extraction Algorithm
Watermarked Video is extracted into number of video shot and particular watermarked frame is selected same as watermark embedding. As shown in Figure 5, Selected 2D frame is converted into 1D pixel string. Using Serial Key same unique PN sequence will be generated which was created for embedding the watermark. Using that PN sequence, watermark size \& watermark logo bits will be extracted from LSB of each pixels of string.1D string of zeroes of watermark size is generated and extracted watermark bits will be set using that PN sequence. At last, 1D string of watermark pixels is reconstructed into 2D image. Extracted image is Watermark logo which was embedded. The embedded watermark can only be extract with correct input password. If it is incorrect, watermark cannot be extract which indicates the improvement in robustness of proposed method as compared to existing LSB algorithm.

\section{IMPLEMENTATION RESULTS AND PERFORMANCE ANALYSIS}

\subsection{Test Video}

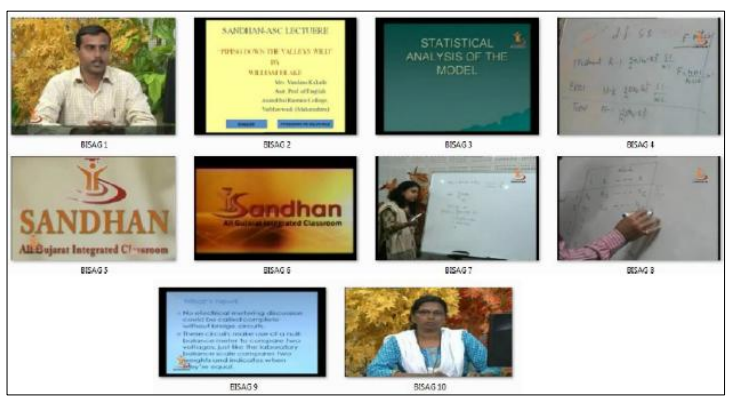

Figure 6: Test Video

Figure 6 shows test videos which are used for implementation and analysis. They are lecture series recorded \& broadcasted by BISAG on channel called SANDHAN. They all are available on YouTube for public use. SANDHAN (All Gujarat Integrated Classroom) provides Distance Education in collaboration with BISAG.

\subsection{Visible Watermark Insertion}

Visible watermark used in this algorithm is Serial Key (Text Watermark). It is 8 digit random number generated using 'rand' function of MATLAB. To the selected bunch of frames, serial key is inserted to bottom left. Figure 7 shows various videos with visible watermark insertion.

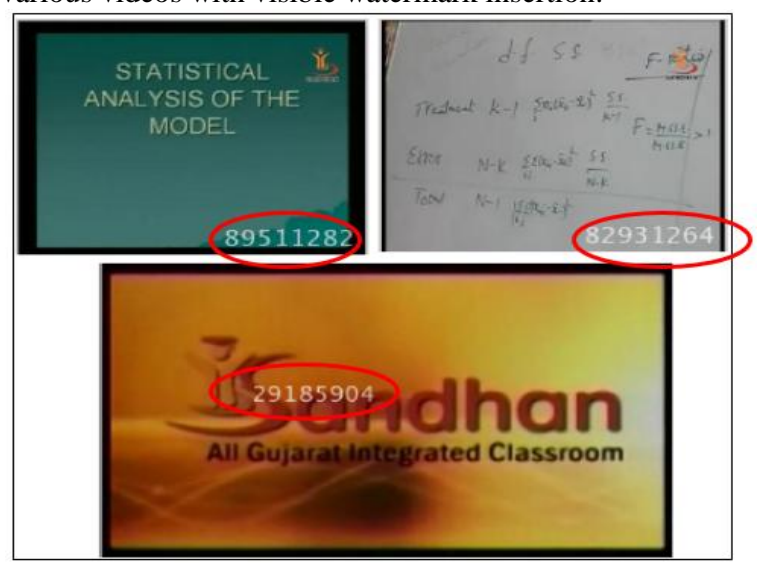

Figure 7. Visible Watermark (Serial Key) Insertion to video 


\subsection{Invisible Watermark Embedding}

Figure 8 shows the result of Watermark Embedding. As shown, first level security is provided by user input password. Frames to be embed the watermark are detected and Watermark is embedded.

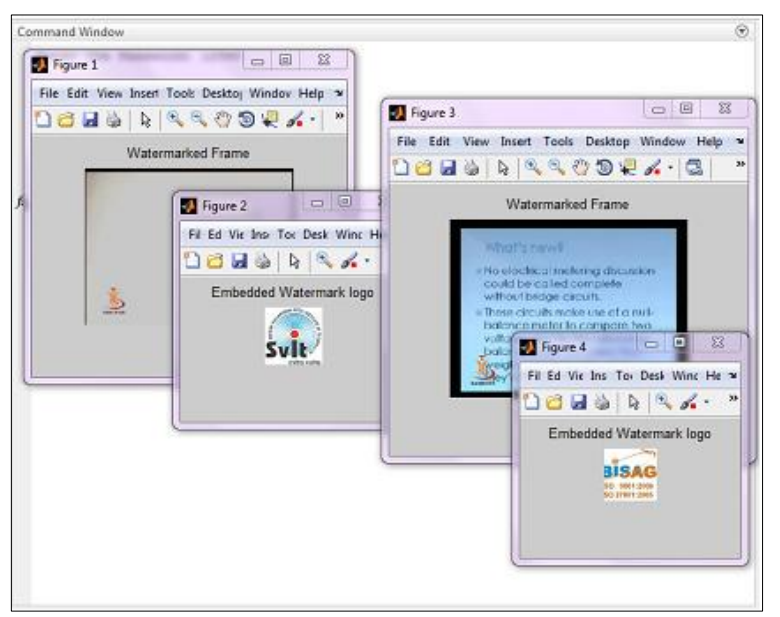

Figure 8. Result for Invisible Watermark Embedding

\section{PERFORMANCE ANALYSIS}

To verify the effectiveness (quality and robustness) of proposed video watermarking technique, several attacks are performed on test videos. Attacks are performed using VSDC Video Editor. Some attacks clearly shows the modification in video as visible serial key shows degradation in it but for some attacks, visible watermark is not detectable or removed. In those cases, modification can be justified by extracting invisible logo.

\subsection{Perceptibility}

Perceptibility measures amount of degradation caused by watermark embedding. Mean Square Error and Peak-Signal-to Noise ratio (PSNR) are used to measure the perceptibility. Less value of MSE and Large value of PSNR indicated low distortion and hence less perceptibility. Thus, Perceptibility should be less. Table. 3 Shows the properties of the videos that are used for experiments.

Table 3. PSNR and MSE of the Watermarked Video

\begin{tabular}{|c|c|c|c|c|c|c|}
\hline Video & $\begin{array}{c}\text { Resolu- } \\
\text { tion }\end{array}$ & $\begin{array}{c}\text { No. } \\
\text { of } \\
\text { Fra- } \\
\text { mes }\end{array}$ & $\begin{array}{c}\text { No. of } \\
\text { Scene } \\
\text { Change } \\
\text { Detected }\end{array}$ & $\begin{array}{c}\text { Execu } \\
\text {-tion } \\
\text { Time } \\
\text { (Sec) }\end{array}$ & MSE & $\begin{array}{c}\text { PSNR } \\
\text { (dB) }\end{array}$ \\
\hline BISAG 1 & $320 \times 240$ & 941 & 2 & 52 & 0.4246 & 51.85 \\
\hline BISAG 2 & $320 \times 240$ & 1725 & 3 & 123 & 0.3541 & 52.64 \\
\hline BISAG 3 & $320 \times 240$ & 3250 & 3 & 173 & 0.4396 & 51.70 \\
\hline BISAG 4 & $320 \times 240$ & 5200 & 10 & 244 & 0.3532 & 52.65 \\
\hline BISAG 5 & $720 \times 480$ & 941 & 2 & 156 & 2.6920 & 43.83 \\
\hline BISAG 6 & $720 \times 480$ & 1725 & 3 & 322 & 1.4590 & 46.49 \\
\hline BISAG 7 & $720 \times 480$ & 3250 & 3 & 637 & 0.7764 & 49.23 \\
\hline BISAG 8 & $720 \times 480$ & 5200 & 10 & 976 & 2,4382 & 44.26 \\
\hline BISAG 9 & $1280 x$ & 1127 & 2 & 494 & 6.4875 & 40.01 \\
\hline BISAG & $1280 x$ & 2067 & 2 & 872 & 5.7687 & 40.52 \\
\hline 10 & 720 & & & & & \\
\hline
\end{tabular}

\subsection{Time Consumption}

It describes the execution time required for watermark embedding. Time is measured by MATLAB 2013a function tic and toc. Below table shows the comparison of execution time required by various other techniques with new approach. The table indicates that watermark embedding time depends both on resolution and number of frames selected to embed the watermark. With the increase in resolution, even for same number of frames required execution times also increases. Table 4 illustrates execution time required by proposed algorithm as compared to other method.

Table 4. Comparison of Proposed Method with others based on PSNR \& Execution time

\begin{tabular}{|c|c|c|c|c|c|c|}
\hline $\begin{array}{c}\text { Video } \\
\text { Frame }\end{array}$ & \multicolumn{2}{|c|}{ DWT } & \multicolumn{2}{c|}{ LSB } & \multicolumn{2}{c|}{$\begin{array}{c}\text { Proposed } \\
\text { Method }\end{array}$} \\
\hline & $\begin{array}{c}\text { Time } \\
\text { (Sec) }\end{array}$ & $\begin{array}{c}\text { PSNR } \\
\text { (dB) }\end{array}$ & $\begin{array}{c}\text { Time } \\
\text { (Sec) }\end{array}$ & $\begin{array}{c}\text { PSNR } \\
\text { (dB) }\end{array}$ & $\begin{array}{c}\text { Time } \\
(\text { Sec) }\end{array}$ & $\begin{array}{c}\text { PSNR } \\
\text { (dB) }\end{array}$ \\
\hline BISAG 1 & 5.42 & 41.44 & 1.85 & 42.11 & $\mathbf{0 . 6 8}$ & $\mathbf{5 1 . 8 5}$ \\
\hline BISAG 2 & 3.59 & 40.11 & 1.79 & 39.92 & $\mathbf{0 . 2 6}$ & $\mathbf{5 2 . 6 4}$ \\
\hline BISAG 3 & 2.38 & 40.45 & 2.16 & 35.13 & $\mathbf{0 . 3 3}$ & $\mathbf{5 1 . 7 0}$ \\
\hline BISAG 4 & 2.42 & 44.67 & 1.82 & 41.89 & $\mathbf{0 . 2 9}$ & $\mathbf{5 2 . 6 5}$ \\
\hline BISAG 5 & 2.45 & 42.67 & 2.66 & 36.90 & $\mathbf{0 . 2 8}$ & $\mathbf{4 3 . 8 3}$ \\
\hline BISAG 6 & 3.11 & 41.90 & 2.92 & 39.23 & $\mathbf{0 . 3 1}$ & $\mathbf{4 6 . 4 9}$ \\
\hline BISAG 7 & 2.5 & 44.67 & 3.06 & 41.45 & $\mathbf{0 . 3 2}$ & $\mathbf{4 9 . 2 3}$ \\
\hline BISAG 8 & 2.64 & 42.56 & 2.89 & 33,17 & $\mathbf{0 . 3 5}$ & $\mathbf{4 4 . 2 6}$ \\
\hline BISAG 9 & 3.67 & 39.67 & 5.58 & 42.19 & $\mathbf{0 . 3 6}$ & $\mathbf{4 0 . 0 1}$ \\
\hline BISAG & 3.24 & 41.90 & 4.50 & 40.12 & $\mathbf{0 . 3 7}$ & $\mathbf{4 0 . 5 2}$ \\
\hline
\end{tabular}

\subsection{Video Specific Attacks}

\subsubsection{Frame Dropping}

As every video exhibits inherent redundancy (Identical video frames), Frame dropping is considered as effective watermark attack which leads little or no damage to video. Video Editors usually cut the portion of some video known as video cutting. This leads to number of frame dropping. In proposed algorithm, if the frames having visible watermark is dropped, invisible watermark is extracted to prove the modification to video. BISAG $8(3 \mathrm{~m} \quad 48 \mathrm{sec})$ video having $720 \times 480$ resolution, total 5200 frames and 10 detected frame by Scene change detection for invisible watermark is used. Invisible watermark logo is inserted into 10 frames where scene change is detected. Table 5 illustrates results of watermark extraction after performing Frame dropping.

Table 5. Results of watermark detection after Frame dropping attack

\begin{tabular}{|c|c|c|c|c|}
\hline $\begin{array}{c}\text { Frame } \\
\text { dropping }\end{array}$ & $\begin{array}{c}\text { Video } \\
\text { Cut(sec) }\end{array}$ & $\begin{array}{c}\text { Total } \\
\text { no. of } \\
\text { frames } \\
\text { Dropped }\end{array}$ & $\begin{array}{c}\text { Visible } \\
\text { watermark } \\
\text { (Detected } \\
\text { ? ) }\end{array}$ & $\begin{array}{c}\text { No. of } \\
\text { Invisible } \\
\text { Watermark } \\
\text { Detected }\end{array}$ \\
\hline $10 \%$ & 21 & 520 & Yes & - \\
\hline $20 \%$ & 41 & 1040 & Yes & - \\
\hline $30 \%$ & 62 & 1560 & No & 8 \\
\hline $40 \%$ & 83 & 2080 & No & 6 \\
\hline $50 \%$ & 104 & 2600 & No & 5 \\
\hline $60 \%$ & 125 & 3120 & No & 3 \\
\hline $70 \%$ & 146 & 3640 & No & 2 \\
\hline $80 \%$ & 166 & 4160 & No & 2 \\
\hline
\end{tabular}




\subsubsection{Frame Insertion}

Same as Frame dropping, Frame adding add some frames to watermarked video. It also means that some portion of video is edited with other video or two videos are merged. Result is same as frame dropping in which till some frame insertion, visible watermark can be detect but after $40 \%$ insertion of video frames, invisible watermark is extracted.

\subsubsection{Frame Averaging}

Frame Averaging is another significant video watermarking attack. Average of multiple frames can remove the visible watermark easily. Frame Averaging is the average of the current and its two nearest neighbor frame. Frame Averaging is defined as :

$F_{k}=\frac{F_{k-1}(i, j)+F_{k}(i, j)+F_{k+1}(i, j)}{3}$

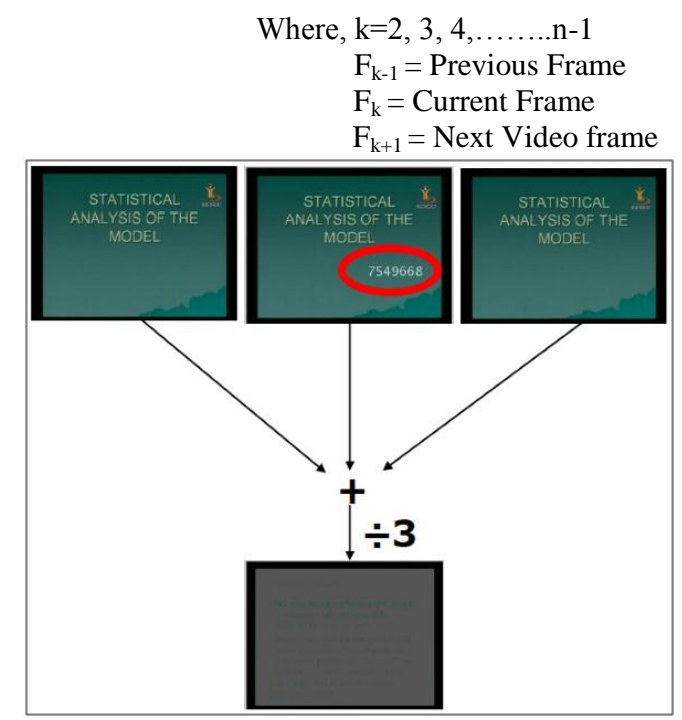

Figure 9. Result of Frame Averaging Attack

\subsubsection{Image Processing Attacks}

Some Image processing attacks are also performed to measure the effectiveness of proposed method. For some attacks, visible watermark (serial key )is identifiable and shows modification done on any video but for some attacks, serial key is removed and hence, modification done onto video is proved by invisible watermark logo extraction.
Table 6. Visible watermark after applying various image processing attacks

\begin{tabular}{|c|c|c|}
\hline Attacks & $\begin{array}{r}\text { Visible Watermark A } \\
\text { attacks }\end{array}$ & ter applying \\
\hline $\begin{array}{l}\text { Contrast } \\
\text { Adjustment }\end{array}$ & $\begin{array}{l}\text { STATISTICAL } \\
\text { ANALYSIS OF THE } \\
\text { MODEL. }\end{array}$ & $\begin{array}{l}\text { Clearly } \\
\text { Detectable }\end{array}$ \\
\hline Grayscale & 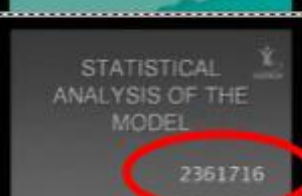 & $\begin{array}{l}\text { Clearly } \\
\text { Detectable }\end{array}$ \\
\hline Sharpening & $\begin{array}{l}\text { STATISTICAL IS } \\
\text { ANALYSIS OF THE } \\
\text { MODE- }\end{array}$ & $\begin{array}{l}\text { Clearly } \\
\text { Detectable }\end{array}$ \\
\hline Max Filtering & 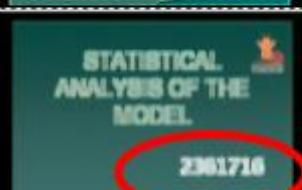 & $\begin{array}{l}\text { Partially } \\
\text { Detectable }\end{array}$ \\
\hline
\end{tabular}

Table 6 shows the identifiable visible watermark after some modification done on video. For some attacks, serial key is removed from video and hence invisible watermark logo extraction is required. Table shows results for such attacks and similarity factor between extracted and reference watermark.

Next, Table 7 describes the similarity between reference and extracted watermark after applying various attacks. As the visible watermark is removed by attacks, invisible watermark is needed to extract. Goof value of SF indicates that extracted watermark is still identifiable after applying attacks. 
Table 7. Extracted Invisible watermark logo after applying attacks

\begin{tabular}{|c|c|c|c|}
\hline \multirow[t]{2}{*}{ Attack } & \multirow[t]{2}{*}{ Visible watermark } & \multicolumn{2}{|c|}{$\begin{array}{c}\text { Invisible } \\
\text { Watermark }\end{array}$} \\
\hline & & $\begin{array}{l}\text { Extracted } \\
\text { Waterma } \\
\text { rk }\end{array}$ & SF \\
\hline $\begin{array}{l}\text { Min } \\
\text { Filtering }\end{array}$ & 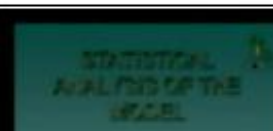 & & 0.619 \\
\hline & Not Detectable & & \\
\hline $\begin{array}{l}\text { Median } \\
\text { Filtering }\end{array}$ & 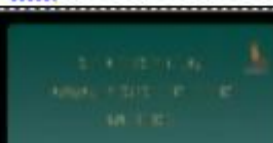 & & 0.758 \\
\hline & Not Detectable & & \\
\hline Blurring & 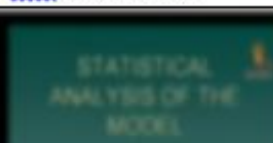 & & 0.719 \\
\hline & Hardly Detectable & & \\
\hline
\end{tabular}

\section{CONCLUSION AND FUTURE SCOPE}

The Proposed Algorithm is useful for the copyright protection of publicly available Educational Videos. Educational lecture series recorded \& broadcasted by various organizations (eg. IITs/ BISAG) requires some means of protection from any illegal attempt to change their identity. Experimental results shows that time required to embedding the watermark is very less as compared to other methods which makes it suitable for watermarking both live as well as recorded videos. High value of PSNR after embedding the watermark also indicates no degradation to the quality of watermarked video. Proposed Algorithm provides two level authentication mechanism on original recorded video.If videos are modified, visible serial key shows degradation in its visibility which proves the illegal attempt to modify the original video. Experimental results shows the robustness of proposed method against various video specific attack, Image processing attacks and Noise attacks but it is not very robust against Geometrical attacks.. If by some method, visible key is removed, invisible watermark logo is used to identify any modification into original video. Modified LSB method embeds the invisible watermark logo using random pattern generated serial key. Proposed algorithm is not robust against Geometrical attacks. In future, the proposed algorithm should be implemented on any open source platform like opencv, scilab, python so that it can be easily implement without the need of any license enabled software like MATLAB. Also, speed of algorithm can be optimized using Parallel Processing.

\section{ACKNOWLEDGMENTS}

Authors would like to thank T.P.Singh, Director, Bhaskaracharya Institute for Space Applications \& Geo- informatics (BISAG, Ghandhinagar) and Dr.Nrhal Chitaliya from Sardar Vallabhbhai Patel Institute of Technology, vasad for aspiring guidance, constructive criticism and valuable suggestion for my research work, as well as to publish this research paper.

\section{REFERENCES}

[1] G. V. A. I. Pitas, "“The use of watermarks in the protection of digital multimedia products" in Proceedings of the IEEE, vol. 87,Issue 7, pp. 1197 - 1207, Jul 1999.

[2] Christoph Busch, Wolfgang Funk, "Digital Watermarking : From concepts to real-time video applications" in IEEE Computer Graphics and Applications, vol.19, Issue 1, pp. 25 - 35, Jan/Feb 1999.

[3] Lee, S.-H. J. Sin-Joo, "A survey of watermarking techniques applied to multimedia" in IEEE International Symposium on Industrial Electronics.(ISIE), vol. 1, pp. 272 - 277, 2001.

[4] Ingemar Cox,Matthew L. Miller,Jeffery A. Bloom, "Digital Watermarking", Morgan Kaufmann, 2001.

[5] Potdar, V.M. ; Song Han ; Chang, E., "A survey of digital image watermarking techniques " in 3rd IEEE International Conference on Industrial Informatics (INDIN'05), pp.709-716,2005.

[6] Rini T Paul, "Review of Robust Video Watermarking Techniques" in IJCA Special Issue on Computational Science - New Dimensions \& Perspectives, vol. 3, pp. 90-95, 2011.

[7] Li Liu, Ling Lu and Daiyuan Peng,' The Design of Secure Video Watermarking Algorithm in Broadcast Monitoring' in IEEE International Conference on Information and Automation ,June 20 -23, 2008, Zhangjiajie, China

[8] Sadik. A.M .Al-Taweel , Putra, Sumari , Hailiza Kamarulhaili, "Digital Video Watermarking Based on 3D-Discrete Wavelet Transform Domain" in IEEE International Conference on Signal and Image Processing Applications, pp.352-356, Malaysia, 2009

[9] S. Al-Taweel and P. Sumari, " Robust Video Watermarking Based On 3D-DWT" in TENCON, IEEE Region 10 Conference, pp.1-6, 2009.

[10] Zamanidoost, S. Mirzakuchaki, R. Atani, Z. Hesabi and M. Ayat, "A novel 3D wavelet-based method for blind digital video watermarking" in IEEE Symposium on Industrial Electronics \& Applications (ISIEA), pp.186189, Penang, 2010

[11] Rama kurupati ,Venkata Rama Prasad,Vaddella,Govardhan Pacha, "Improved Imperceptibility of watermarked Images using DWT \& DCT Transform" in National Conference on Imaging,Computing,Object \& Mining (ICOM'10), Tamil Nadu,India, May,2010.

[12] Kamble, S. ; Maheshkar, V. ; Agarwal, S. ; Srivastava, V.K., "DWT-based multiple watermarking for privacy and security of digital images in e-commerce" in IEEE International Conference on Multimedia, Signal Processing and Communication Technologies (IMPACT), pp.224-227, 2011. 
[13] Amit Joshi, Vivekanand Mishra and R. M. Patrikar, "Real Time Implementation of Digital Watermarking Algorithm for Image and Video Application" in Watermarking, vol. 2, pp. 67-90, Dr. Mithun Das Gupta, Ed., Intech, 2012.

[14] Tabassum, T. ; Islam, S.M.M., "A digital video watermarking technique based on identical frame extraction in 3-Level DWT" in 15th IEEE International Conference on Computer and Information Technology (ICCIT),pp.101-106, 2012.

[15] Manpreet kaur, Sonia Jindal, Sunny behal, "A Study of Digital image watermarking" in International Journal of Research in Engineering \& Applied Sciences , Vol.2, Issue 2, pp.126-136, Feb 2012.
[16] Amit Kumar Singh, Nomit Sharma, Mayank Dave, Anand Mohan, "A Novel Technique for Digital Image Watermarking in Spatial Domain" in 2nd IEEE International Conference on Parallel, Distributed and Grid Computing, pp. 497-501, 2012.

[17] Tabassum, T. ; Islam, S.M.M., "A digital video watermarking technique based on identical frame extraction in 3-Level DWT" in 15th IEEE International Conference on Computer and Information Technology (ICCIT),pp.101-106, 2012.

[18] Venugopala, P.S. ; Sarojadevi, H. ; Chiplunkar, N.N. ; Bhat, V, "Video Watermarking by Adjusting the Pixel Values and Using Scene Change Detection," in Signal and Image Processing (ICSIP), 2014 Fifth International Conference on, 2014. 